\title{
Effects of the Kampo Formula Tokishakuyakusan on Headaches and Concomitant Depression in Middle-Aged Women
}

\author{
Masakazu Terauchi, ${ }^{1}$ Shiro Hiramitsu, ${ }^{2}$ Mihoko Akiyoshi, ${ }^{2}$ Yoko Owa, ${ }^{2}$ Kiyoko Kato, \\ Satoshi Obayashi, ${ }^{2}$ Eisuke Matsushima, ${ }^{3}$ and Toshiro Kubota ${ }^{2}$ \\ ${ }^{1}$ Department of Women's Health, Tokyo Medical and Dental University, Yushima 1-5-45, Bunkyo, Tokyo 113-8510, Japan \\ ${ }^{2}$ Department of Obstetrics and Gynecology, Tokyo Medical and Dental University, Yushima 1-5-45, Bunkyo, Tokyo 113-8510, Japan \\ ${ }^{3}$ Department of Psychosomatics, Tokyo Medical and Dental University, Yushima 1-5-45, Bunkyo, Tokyo 113-8510, Japan \\ Correspondence should be addressed to Masakazu Terauchi; teragyne@tmd.ac.jp
}

Received 11 September 2013; Revised 27 December 2013; Accepted 27 December 2013; Published 4 February 2014

Academic Editor: Shun-Wan Chan

Copyright (C) 2014 Masakazu Terauchi et al. This is an open access article distributed under the Creative Commons Attribution License, which permits unrestricted use, distribution, and reproduction in any medium, provided the original work is properly cited.

Objectives. To identify the correlates of headaches in middle-aged women and investigate the effects of Tokishakuyakusan (TJ-23), a formula of traditional Japanese herbal therapy Kampo, on headache and concomitant depression. Methods. We examined crosssectionally the baseline records of 345 women aged 40-59 years who visited our menopause clinic. Among them, 37 women with headaches were treated with either hormone therapy (HT) or TJ-23; the data of these women were retrospectively analyzed to compare the effects of the treatment. Results. The women were classified into 4 groups on the basis of their headache frequency, and no significant intergroup differences were noted in the physical or lifestyle factors, except age. Multiple logistic regression analysis revealed that the significant contributors to the women's headaches were their age (adjusted OR 0.92 (95\% CI 0.88-0.97)) and their depressive symptoms (adjusted OR 1.73 (95\% CI 1.39-2.16)). Compared to women treated with HT, women treated with TJ-23 reported relief from headaches (65\% versus $29 \%$ ) and concomitant depression (60\% versus $24 \%$ ) more frequently. Improvement in the scores of headaches and depression correlated significantly with TJ-23 treatment. Conclusions. Headache in middle-aged women is significantly associated with depression; TJ-23 could be effective for treating both of these symptoms.

\section{Introduction}

Headache is one of the most common symptoms observed in community and primary care settings, as exemplified by a study revealing that $40 \%$ of the Nordic women in rural communities experience it, indicating that it is more frequent than other somatic symptoms [1]. As expected, headache is included in most of the inventories of menopausal symptom checklists, although the influence of menopause on women's headache depends on the subtypes of headache, such as tension-type headache (TTH) and migraine, which are the 2 most common ones. More than two-thirds of women with TTH report unchanged or worse status of headache after menopause [2]; on the other hand, migraine improves after menopause, and one of the triggers of migraine is postulated to be withdrawal of estrogen [3]. The 2 major types of primary headache mentioned above are, however, often difficult to differentiate in clinical practice and are summarized simply as "headaches" in most of the menopause assessment scales, such as Greene Climacteric Scale [4] and Women's Health Questionnaire [5].

Headache has been reported to be associated with psychological symptoms. A large-scale study on the Nordic community revealed that depression and anxiety were significantly associated with migraine and nonmigrainous headache and that the former was more strongly associated with psychological symptoms than the latter [6]. A correlation between headache and depression in middle-aged women was also noted in studies conducted in the United States [7] and Japan [8].

Kampo, a Japanese subdivision of traditional East Asian medicines, was founded in the 6th century as a local adaptation of the Chinese herbal medicine. Kampo is a more pragmatic approach to complementary and alternative 
medicine than the Chinese herbal medicine. In the Chinese herbal medicine, a medical practitioner confirms the treatment principle according to "Zheng" (the clinical diagnosis determined on the basis of an analysis of the patient's history, symptoms, and signs) and then mixes $10-15$ herbs to create a specific formula for each patient. In Kampo, a practitioner chooses the best formula for a patient from approximately 150 ready-to-use formulae-each of which is typically composed of 5-9 herbs-on the basis of the patient's "Sho" (pattern of symptoms) $[9,10]$.

One of the most frequently used Kampo formulae in the management of menopausal symptoms in Japan is Tokishakuyakusan (TJ-23), which is an extract of granules made from a herb mixture of Peony root, Atractylodes lancea rhizome, Alisma rhizome, Poria sclerotium, and Cnidium officinale rhizome and the Japanese Angelica root. It is indicated for various symptoms, including headache, in patients who have generally weak muscles, are easily fatigued, and whose waist and lower limbs are susceptible to cold.

In the present study, we sought to determine the prevalence of headache and its correlates in the Japanese periand postmenopausal women and investigate the effect of the Kampo formula TJ-23 on their headache and concomitant depression.

\section{Materials and Methods}

In this retrospective study, we examined the medical records of the same study population examined in our previous study $[8,11-16]$. Specifically, we analyzed the records of 345 Japanese peri- and postmenopausal women (age: 4059 years) who had been enrolled in the Systematic Health and Nutrition Education Program (SHNEP) conducted at the Menopause Clinic of the Tokyo Medical and Dental University Hospital between February 2006 and December 2010. All the middle-aged women enrolled in this program had been referred to our clinic for the treatment of their menopausal symptoms and had provided informed consent for participation. Before beginning our investigation, we obtained approval for the study protocol from the Tokyo Medical and Dental University Review Board. All procedures in the study were in accordance with the Declaration of Helsinki.

The goals of SHNEP were to improve the general health status by prescribing appropriate medications after a physician's assessment and providing advice on diet and exercise regimens after lifestyle assessment by nutritionists.

The mean \pm SD age of the 345 participants was $50.9 \pm 4.5$ years. The participants were classified as being in menopause transition or as postmenopausal, on the basis of an analysis of their menstrual cycles. A woman was defined as being in menopause transition if she had had a menstrual period within the past 12 months but had missed a period or if she had irregular cycles in the past 3 months. Women were classified into the postmenopausal group in the absence of a menstrual period in the past 12 months [17]. At their initial visit, the women were interviewed by physicians and nutritionists and provided data on their menopausal symptoms and quality of life during the past month by answering the Menopausal Health-Related Quality of Life (MHR-QOL) questionnaire, which has been developed and validated at our clinic $[8,11-16]$. The MHR-QOL questionnaire is a modification of the Women's Health Questionnaire developed by Hunter $[5,18]$ and contains 38 items scored on a 4-point or a binary scale, covering 4 major domains (physical health, mental health, life satisfaction, and social involvement) of a woman's health during menopausal transition.

The items used to assess the 2 domains of interest in the current study, namely, physical health and mental health, are shown in Table 1. As shown, the physical health domain comprised 9 items that assess somatic symptoms, including headaches and vasomotor symptoms, and the mental health domain comprised 12 items that assess depressed mood, anxiety and fears, and sleep disturbances. For convenience, the scoring system used in the present study is the reverse of that used for the QOL questionnaire in our clinical practice; that is, the higher the scores for the 9 evaluated items, the poorer the physical and mental functioning: 0-1 time a month, 0 point; 1 - 2 times a week, 1 point; $3-4$ times per week, 2 points; and almost every day, 3 points.

Data were recorded on the following physical characteristics of the women enrolled in the SHNEP: height $(\mathrm{cm})$; body weight (kg); body mass index (BMI), which was calculated as weight $(\mathrm{kg}) / \mathrm{height}^{2}\left(\mathrm{~m}^{2}\right)$; waist circumference $(\mathrm{cm})$; hip circumference $(\mathrm{cm})$; waist-hip ratio, which was calculated as waist circumference/hip circumference; body fat percentage (\%); lean body mass $(\mathrm{kg})$; and body water mass $(\mathrm{kg})$, with the latter 3 being measured using the body composition analyzer MC-190EM (Tanita, Tokyo, Japan). Systolic and diastolic blood pressure, pulse rate, cardio-ankle vascular index (CAVI), as an indicator for atherosclerosis, and anklebrachial index (ABI), as an indicator for peripheral vascular disease, were measured using VS-1000 (Fukuda Denshi, Tokyo, Japan) [19].

The following lifestyle characteristics were also assessed: amount of daily caffeinated beverage consumption (more than 3 cups, 1-3 cups, none), frequency of alcohol consumption (daily, sometimes, never), and the habit of smoking (more than 20 cigarettes per day, 1-20 cigarettes per day, none).

Among the 182 participants who reported having headaches once or more than once a week at their first visits, 37 were treated with either hormone therapy $(\mathrm{HT}, N=17)$ or TJ-23 $(N=20)$; these 37 patients were selected for further analysis. Estrogens included in the HT regimens used for these participants were either $0.625 \mathrm{mg} /$ day of conjugated estrogen $(N=12), 50 \mu \mathrm{g} /$ day of estradiol via a transdermal patch $(N=3)$, and $1.0 \mathrm{mg}$ of oral micronized estradiol $(N=2)$. For the women with an intact uterus $(N=8)$, medroxyprogesterone acetate was used either continuously or sequentially. TJ-23 was prescribed in the form of extract granules (7.5 g/day) comprising Peony root (9.7\%), A. lancea rhizome, Alisma rhizome, P. sclerotium, C. officinale rhizome (7.3\%), and Japanese Angelica root (TJ-23, Tsumura, Tokyo). We excluded the following subjects from the analysis: (1) women who were prescribed nonsteroidal anti-inflammatory drugs or triptans to treat their headaches or migraines; (2) 
TABLE 1: Prevalence of each item in the physical and mental health domains in the Menopausal Health Related Quality of Life (MHR-QOL) questionnaire $(\%, N=345)$.

\begin{tabular}{|c|c|c|c|c|}
\hline & $0-1$ time a month & 1-2 times a week & 3-4 times per week & Almost every day \\
\hline \multicolumn{5}{|l|}{ Physical health domain } \\
\hline Nausea & 78.5 & 13.4 & 2.3 & 5.8 \\
\hline Dizziness & 65.4 & 22.2 & 5.2 & 7.3 \\
\hline Numbness & 60.4 & 13.5 & 6.7 & 19.4 \\
\hline Muscle and joint pains & 12.8 & 18.6 & 13.3 & 55.4 \\
\hline Tiredness & 14.5 & 21.2 & 15.1 & 49.3 \\
\hline Headaches & 47.2 & 27.5 & 11.6 & 13.6 \\
\hline Frequent urination & 52.5 & 15.2 & 8.2 & 24.2 \\
\hline Hot flushes & 45.3 & 18.0 & 15.1 & 21.5 \\
\hline Night sweats & 55.4 & 17.4 & 10.4 & 16.8 \\
\hline \multicolumn{5}{|l|}{ Mental health domain } \\
\hline Loss of interest in things & 51.9 & 18.1 & 13.1 & 16.9 \\
\hline Lack of enjoyment & 48.7 & 22.0 & 11.9 & 17.4 \\
\hline Low energy & 34.9 & 27.3 & 14.2 & 23.5 \\
\hline Depressed mood & 30.8 & 27.6 & 15.7 & 25.9 \\
\hline Poor memory & 27.5 & 32.5 & 20.6 & 19.4 \\
\hline Difficulty in concentration & 31.6 & 29.3 & 20.3 & 18.8 \\
\hline Frightened/panicky feelings & 46.2 & 22.7 & 15.4 & 15.7 \\
\hline Feel tense/wound up & 37.5 & 24.1 & 17.2 & 21.2 \\
\hline Dissatisfaction with sexual relationship & 85.0 & 6.8 & 2.9 & 5.3 \\
\hline Difficulty in initiating sleep & 46.4 & 18.8 & 11.6 & 23.2 \\
\hline Nonrestorative sleep & 34.5 & 21.4 & 13.6 & 30.4 \\
\hline Low self-esteem & 39.1 & 21.0 & 12.0 & 28.0 \\
\hline
\end{tabular}

women who were prescribed medications other than HT or TJ-23 alone to treat menopausal symptoms; and (3) women who were prescribed any psychotropic drugs, such as antidepressants, anxiolytics, and hypnotics.

All statistical analyses were performed with SAS 9.2 (SAS Institute, Cary, NC, USA). We performed statistical analyses using one-way analysis of variance, Fisher's exact test, multiple logistic regression with stepwise variable selection procedure, unpaired $t$-test, and the Kruskal-Wallis test. Statistical significance was defined as $P<0.05$.

\section{Results}

Of the 345 women studied, 160 (46.4\%) were in menopause transition and 185 (53.6\%) were postmenopausal.

The prevalence of each item in the physical and mental health domains of the MHR-QOL questionnaire among the evaluated women is shown in Table 1 . The percentages of women who experienced headaches $0-1$ time a month, 1-2 times a week, 3-4 times per week, or almost every day were $47.2 \%, 27.5 \%, 11.6 \%$, and $13.6 \%$, respectively. Headache ranked 13 th among the 21 items, assessed according to the percentage of women who experienced the symptom more than once a week $(52.8 \%)$.

First, we compared the baseline characteristics of the 4 groups of women classified on the basis of their headache frequency (Table 2). There were no significant intergroup differences in any of the physical or lifestyle factors, except age. The average age of the women who had headaches almost every day was significantly less (by almost 2 years) than those of the women who experienced the symptom once a month or less frequently ( $P<0.05$, Tukey's test). Additionally, no significant intergroup differences were noted in the ratio of the women in menopause transition to those who were postmenopausal.

Second, we examined the correlations between headaches and other symptoms, namely, vasomotor, depressive, anxious, and insomnia. The scores for the 2 vasomotor symptoms in the MHR-QOL questionnaire (hot flushes and night sweats) were averaged to generate a vasomotor score. Likewise, the depression score, anxiety score, and insomnia score were calculated from the averages of the scores of 4 depressive symptoms ("loss of interest in things," "lack of enjoyment," "low energy," and "depressed mood"), 2 anxious symptoms ("frightened/panicky feelings" and "feel tense/wound up"), and 2 insomnia symptoms scores ("difficulty in initiating sleep" and "nonrestorative sleep"), respectively. To determine whether these symptoms were associated with headaches in middle-aged women, we performed a multiple logistic regression analysis by using the presence of headaches once a week or more as the dependent variable and age and the scores for vasomotor symptoms, depression, anxiety, and insomnia as independent variables. Table 3 shows the crude and adjusted ORs for assessing the strength of the relationship between each factor and headaches. Although all the variables 
TABLE 2: Baseline characteristics of the study participants by groups classified on the basis of their headache frequency $(N=345)$.

\begin{tabular}{|c|c|c|c|c|c|}
\hline & $\begin{array}{l}0-1 \text { time a month } \\
(N=163)\end{array}$ & $\begin{array}{l}\text { 1-2 times a week } \\
(N=95)\end{array}$ & $\begin{array}{l}\text { 3-4 times per week } \\
\quad(N=40)\end{array}$ & $\begin{array}{l}\text { Almost every day } \\
(N=47)\end{array}$ & $P$ value \\
\hline Age (years) & $51.6 \pm 4.5$ & $50.1 \pm 4.3$ & $51.1 \pm 4.7$ & $49.7 \pm 4.1$ & 0.012 \\
\hline \multicolumn{6}{|l|}{ Menopause status (\%) } \\
\hline Menopause transition & 44 & 48 & 53 & 47 & \multirow{2}{*}{0.737} \\
\hline Postmenopausal & 56 & 52 & 48 & 53 & \\
\hline Height $(\mathrm{cm})$ & $156.2 \pm 10.9$ & $158.0 \pm 4.6$ & $156.7 \pm 4.8$ & $156.7 \pm 4.6$ & 0.406 \\
\hline Body weight (kg) & $52.8 \pm 8.1$ & $53.0 \pm 7.9$ & $53.2 \pm 11.3$ & $54.3 \pm 10.7$ & 0.809 \\
\hline Body mass index $\left(\mathrm{kg} \cdot \mathrm{m}^{-2}\right)$ & $21.5 \pm 3.3$ & $21.3 \pm 3.1$ & $21.6 \pm 3.6$ & $22.1 \pm 4.2$ & 0.639 \\
\hline Waist circumference $(\mathrm{cm})$ & $77.9 \pm 9.3$ & $77.4 \pm 8.5$ & $79.0 \pm 10.9$ & $78.4 \pm 11.4$ & 0.824 \\
\hline Hip circumference $(\mathrm{cm})$ & $91.4 \pm 6.1$ & $90.8 \pm 5.5$ & $91.3 \pm 7.8$ & $91.9 \pm 7.5$ & 0.819 \\
\hline Waist-hip ratio & $0.85 \pm 0.06$ & $0.85 \pm 0.06$ & $0.86 \pm 0.07$ & $0.85 \pm 0.07$ & 0.771 \\
\hline Body fat $(\%)$ & $26.7 \pm 7.4$ & $27.0 \pm 7.9$ & $28.5 \pm 7.9$ & $27.0 \pm 8.9$ & 0.733 \\
\hline Muscle mass (kg) & $35.6 \pm 2.8$ & $36.1 \pm 2.8$ & $36.0 \pm 3.1$ & $36.0 \pm 2.8$ & 0.648 \\
\hline Body water mass (kg) & $27.2 \pm 2.8$ & $27.4 \pm 2.9$ & $27.6 \pm 3.1$ & $27.6 \pm 3.1$ & 0.885 \\
\hline Systolic pressure (mmHg) & $124.3 \pm 16.5$ & $122.5 \pm 12.4$ & $127.7 \pm 19.3$ & $123.8 \pm 14.1$ & 0.382 \\
\hline Diastolic pressure $(\mathrm{mmHg})$ & $79.9 \pm 11.4$ & $79.0 \pm 8.8$ & $80.9 \pm 12.4$ & $79.3 \pm 11.6$ & 0.801 \\
\hline Pulse rate $\left(\mathrm{min}^{-1}\right)$ & $62.7 \pm 10.4$ & $61.0 \pm 12.2$ & $62.2 \pm 8.2$ & $64.6 \pm 10.2$ & 0.297 \\
\hline Cardio-ankle vascular index (CAVI) & $7.54 \pm 0.68$ & $7.40 \pm 0.60$ & $7.48 \pm 0.75$ & $7.55 \pm 0.74$ & 0.479 \\
\hline Ankle-brachial index (ABI) & $1.11 \pm 0.06$ & $1.12 \pm 0.06$ & $1.11 \pm 0.06$ & $1.11 \pm 0.07$ & 0.930 \\
\hline \multicolumn{6}{|l|}{ Alcohol consumption (\%) } \\
\hline Daily & 15 & 10 & 11 & 9 & \multirow{3}{*}{0.711} \\
\hline Sometimes & 22 & 30 & 26 & 22 & \\
\hline None & 63 & 60 & 63 & 69 & \\
\hline \multicolumn{6}{|l|}{ Smoking (\%) } \\
\hline$\geq 20$ cigarettes/day & 4 & 3 & 5 & 7 & \multirow{3}{*}{0.821} \\
\hline$<20$ cigarettes/day & 9 & 12 & 5 & 11 & \\
\hline None & 87 & 85 & 89 & 82 & \\
\hline \multicolumn{6}{|l|}{ Caffeinated beverage consumption (\%) } \\
\hline$\geq 3$ cups/day & 70 & 68 & 68 & 53 & \multirow{3}{*}{0.365} \\
\hline$<3$ cups/day & 22 & 23 & 29 & 38 & \\
\hline None & 8 & 9 & 3 & 9 & \\
\hline
\end{tabular}

Data are expressed as the mean \pm standard deviation or percentage.

The $P$ values were derived from one-way analysis of variance or Fisher's exact test.

TABlE 3: Contribution of age, vasomotor symptoms, depression, anxiety, and insomnia to headaches in peri- and postmenopausal women $(N=345)$

\begin{tabular}{lccc}
\hline & Crude OR $(95 \% \mathrm{CI})$ & $P$ value & Adjusted OR (95\% CI) \\
\hline Age & $0.93(0.89-0.98)$ & 0.0038 & $0.92(0.88-0.97)$ \\
Vasomotor score & $1.31(1.06-1.63)$ & 0.0136 & $1.73(1.39-2.16)$ \\
Depression score & $1.69(1.36-2.10)$ & $<0.0001$ & $<0.0019$ \\
Anxiety score & $1.51(1.23-1.86)$ & $<0.0001$ & \\
Insomnia score & $1.40(1.16-1.70)$ & 0.0006 & \\
\hline
\end{tabular}

OR: odds ratio; CI: confidence interval.

included were significantly associated with headaches in the univariate logistic regression analysis, subsequent multiple logistic regression analysis with stepwise variable selection procedure revealed that only age (adjusted OR, 0.92; 95\% CI, $0.88-0.97 ; P=0.0019$ ) and depression (adjusted OR, 1.73; 95\% CI, 1.39-2.16; $P<0.0001)$ were significantly associated with headaches after adjustment. The highest condition index of 1.04 suggested the absence of collinearity among the 2 variables.

The baseline characteristics of the 37 study subjects treated with HT $(N=17)$ or TJ-23 $(N=20)$ for headaches are shown in Table 4 . There were no significant differences 
TABLE 4: Baseline characteristics of the study subjects $(N=37)$.

\begin{tabular}{lccc}
\hline & HT $(n=17)$ & TJ-23 $(n=20)$ & $P$ value \\
\hline Age (years) & $48.9(3.6)$ & $50.7(5.4)$ & \\
Frequency of headaches (\%) & 71 & 50 & 0.249 \\
$\quad$ 1-2 times a week & 18 & 25 & 0.531 \\
3-4 times per week & 12 & $1.38(1.19)$ & 0.756 \\
$\quad$ Almost every day & $1.26(1.16)$ & $1.54(0.75)$ & 0.124 \\
Vasomotor score & $1.24(0.68)$ & $1.48(0.85)$ & 0.439 \\
Depression score & $1.29(1.05)$ & $1.70(1.20)$ & 0.731 \\
Anxiety score & $1.56(1.16)$ & & \\
Insomnia score & & & \\
\hline
\end{tabular}

Data are expressed as the mean (standard deviation) or percentage.

$P$ values were derived from the unpaired $t$-test (age), Fisher's exact test (frequency of headaches), or Kruskal-Wallis test (symptom scores).

between the groups regarding the age, headache frequency, or baseline symptom scores.

The percentages of women whose symptom scores decreased after a follow-up period of $147 \pm 56$ (mean \pm $\mathrm{SD})$ days were compared. Significantly more women in the TJ-23 group reported relief from headaches and depression than those in the HT group (headaches, $65 \%$ versus $29 \%$; depression, $60 \%$ versus $24 \%$ ); however, there were no significant intergroup differences in the improvement of vasomotor symptoms, anxiety, and insomnia (Figure 1).

Finally, an analysis of the association between improvement in headaches and depression in the TJ-23 group showed a significant correlation between the changes in the headache and depression scores with TJ-23 treatment (Figure 2).

\section{Discussion}

In this study, we aimed to identify the correlates of headaches in middle-aged women on the basis of a cross-sectional analysis of the records of 345 women participating in a health and education program. We also performed a retrospective cohort analysis to compare the effects of HT and the Kampo formula TJ-23 on the headaches and concomitant depression in these women.

A comparison of the physical and lifestyle factors among the groups of participants classified by their headache frequency suggested that younger women had more headaches than those who are older and that the symptom was not necessarily more frequent among women in menopause transition than in those who are postmenopausal. A classic article by Neugarten and Kraines [20] on menopausal symptoms indicated that the percentage of headaches in women aged over 55 years was remarkably lesser than that in women aged under 44 years, although the effect of menopause is difficult to ascertain because the study compared "menopausal" women with "pre- or postmenopausal" ones belonging to the same age group of 45-54 years. Recently, Berecki-Gisolf closely dissected the associations of age and menopause with the symptoms of middle-aged women in a large-scale prospective cohort study; they reported that age and postmenopause status, rather than perimenopause or menopause transition and premenopause, are significant negative contributors to headaches [21]. In our study, slightly more perimenopausal women $(47 \%)$ were present in the group with headache almost every day than in the group with headaches at a frequency of once a month or less (44\%). A larger sample size may have afforded a statistically significant intergroup difference.

Headache is known to be associated with psychological symptoms and is included in several somatization screening measures, such as Patient Health Questionnaire (PHQ)-15, World Health Organization (WHO)-Social Security Disability (SSD), and Symptom Checklist (SCL)-12 [22]. In the current study, the presence of headaches once a week or more frequently was found to be associated with depression but not with vasomotor symptoms, anxiety, or insomnia after adjustment. The association between depression and headaches was also confirmed in our previous study [8] and a US population-based study of middle-aged women [7]. On the other hand, a large-scale community study in Norway (HUNT-II) revealed that the Hospital Anxiety and Depression scale scores were significantly associated with the presence of migraine and nonmigrainous headaches [6]. The findings of the Nordic study in which the correlation with headaches is stronger for anxiety than for depression are not consistent with our finding; this discrepancy may be partly attributable to the differences in the sample backgrounds, such as age, ( $\geq 20$ versus $40-60$ years), sex (both versus female only), ethnicity (Nordic versus Japanese), and settings (community versus clinical).

The effects of ovarian sex steroids and menopause on headaches appear to differ from the type of headache. TTH is slightly more prevalent in women than in men, and the average of the reported male-to-female ratios is 1.30 [2]. This difference is not recognized until children reach puberty, thereby suggesting the involvement of sex steroids in the pathogenesis of TTH [2], although one report suggests that TTH status remains unchanged or becomes worse in $70 \%$ of women with this type of headache after menopause [23]. The sex difference is more marked in the case of migraine: the cumulative lifetime incidence for women is $43 \%$, while that for men is $18 \%$ [3]. Migraine is known to be affected by hormonal fluctuations, with the effect of estrogen withdrawal being predominant during the perimenopausal period [3]. With the absence of fluctuations 


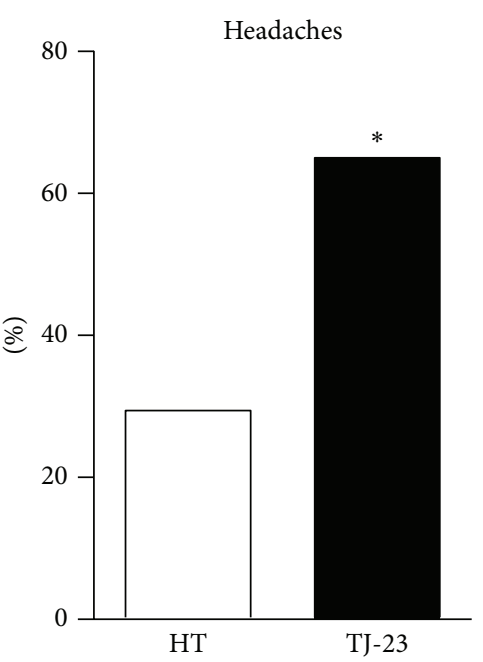

(a)

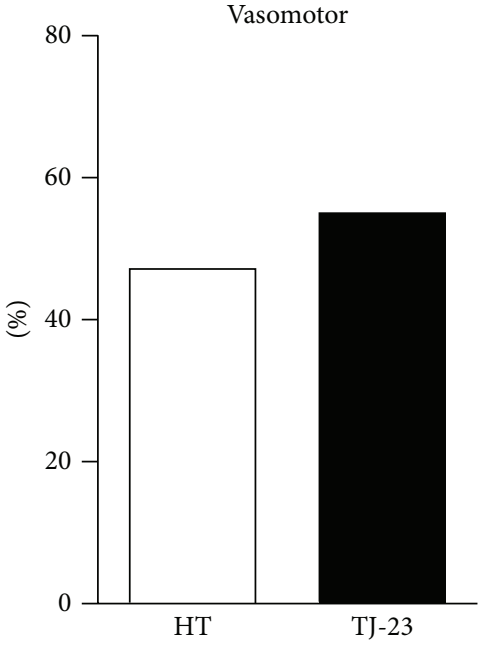

(b)

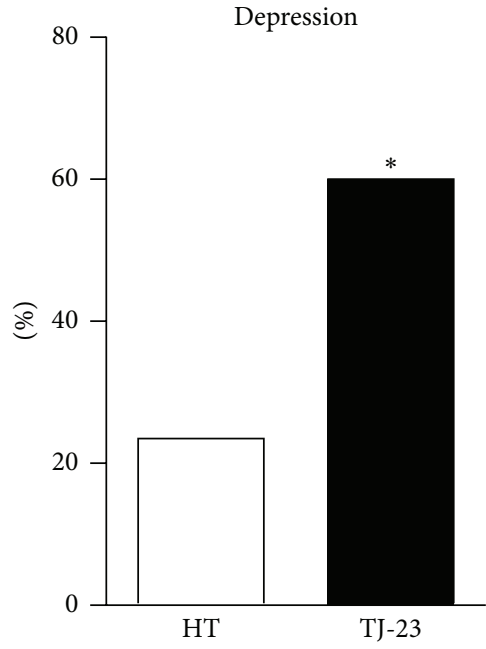

(c)

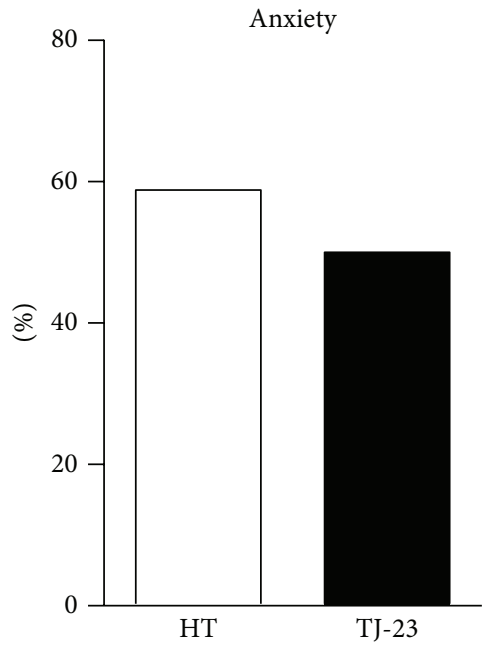

(d)

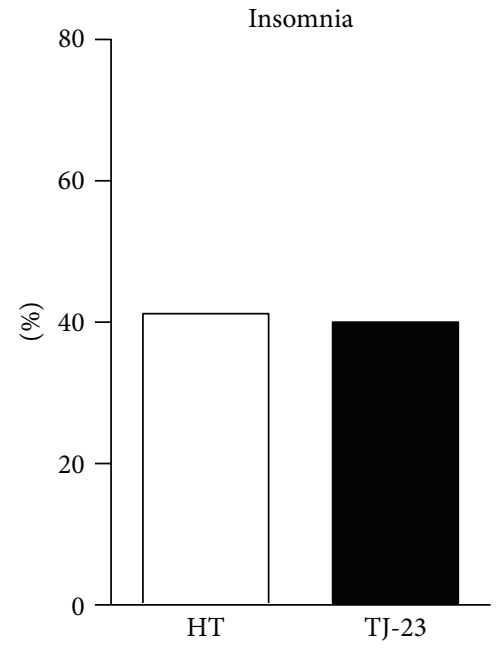

(e)

FIGURE 1: The percentage of women whose symptom scores decreased after treatment with hormone therapy (HT) or Tokishakuyakusan (TJ-23) ${ }^{*} ; P<0.05$ versus HT.

in sex hormone levels, the percentage of women reporting migraine after menopause is reduced, as demonstrated by the Penn ovarian aging study [24]. Although HT should be theoretically effective for perimenopausal migraine controlling the hormonal fluctuation, the reports are inconsistent. For example, a large population-based study showed a significant association between migraine and nonmigrainous headache with the current use of systemic HT [25]. In the present study, the percentages of women receiving HT whose headache improved, did not change, or worsened were $29 \%$, 59\%, and $12 \%$, respectively, which were even better than the percentages indicated in Mueller's report on migraineurs only [26].

Complementary and alternative medicines (CAM) are extensively used worldwide, especially by women, middleaged individuals, and people with chronic diseases or poor overall health [27]. In the early 2000 s, approximately $50 \%$ of all middle-aged women in Western countries used CAM to alleviate menopausal symptoms $[27,28]$. The current percentage may be even higher, considering the sustained decline in HT use among this population since the publication of the Women's Health Initiative results $[29,30]$. A recent review by Adams revealed that a substantial proportion of people with headache and migraine use CAM and find it effective [31].

TJ-23 is one of the most frequently used Kampo formulae for the management of menopausal symptoms in Japan. According to the package insert, TJ-23 is indicated for the relief of climacteric disturbance (dull headache, headache, dizziness, shoulder stiffness, etc.) of patients who have generally weak muscles, are easily fatigued, and whose waist and lower limbs are susceptible to cold. The extracts of Ligusticum chuanxiong, a species closely related to the TJ-23 component Cnidium officinale, have long been used as an angesic and were shown to alleviate headache [32] and migraine [33] in animal models. Recent reports also indicate that the Chinese 


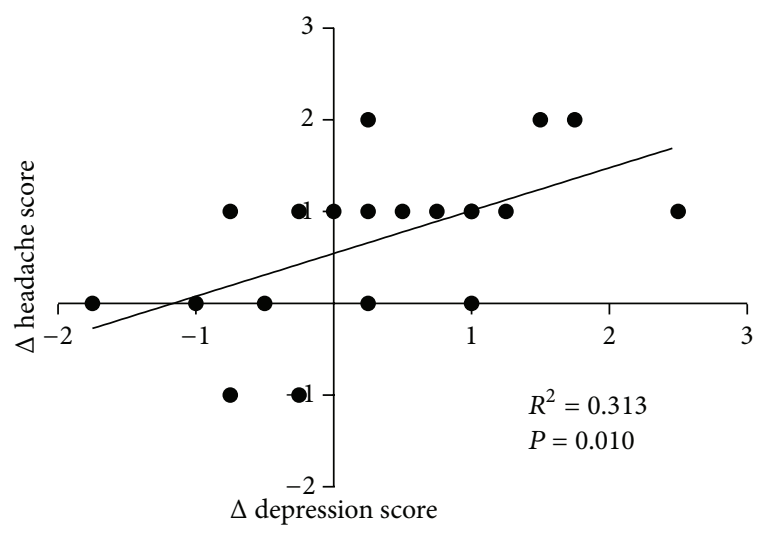

FIGURE 2: The association between the improvements in headaches and depression in the TJ-23 group.

equivalent of TJ-23, Danggui-Shaoyao-San, exerts antidepressant effects in animal models possibly by suppressing the expression of arginine vasopressin in the pituitary and hypothalamus [34]. The improvements in both headache and depression induced by TJ-23 could also be attributed to these pharmacological mechanisms. Furthermore, considering the association between headache and depression, it appears that the relieving effect of TJ-23 on headache may have helped improve the women's mood and vice versa [8].

One of the major limitations of our study is that our questionnaire and the subsequent analysis did not differentiate among the headache subtypes. Headaches have been clearly categorized by the International Headache Society as per the International Headache Classification system, with the intention of developing evidence-based treatment strategies for the patients. However, the accurate categorization of the headache subtype is sometimes difficult in clinical practice and may not be as useful to treatment as the proper assessment of the severity of the condition [35]. Furthermore, the retrospective design of the current study might have introduced a disparity between the treatment groups although no significant intergroup differences were observed in the background characteristics. Finally, further prospective studies worldwide using TJ-23 or Tokishakuyakusan are warranted to confirm the findings obtained from the current retrospective analysis of the limited number of subjects.

\section{Conclusions}

In conclusion, headaches in middle-aged women are significantly associated with depression. The Kampo formula TJ23 could be an effective treatment for the women with both symptoms.

\section{Conflict of Interests}

The authors declare that there is no conflict of interests regarding the publication of this paper.

\section{References}

[1] T. T. Haug, A. Mykletun, and A. A. Dahl, "The association between anxiety, depression, and somatic symptoms in a large population: the HUNT-II study," Psychosomatic Medicine, vol. 66, no. 6, pp. 845-851, 2004.

[2] D. Lieba-Samal and C. Wöber, "Sex hormones and primary headaches other than migraine," Current Pain and Headache Reports, vol. 15, no. 5, pp. 407-414, 2011.

[3] E. A. MacGregor, "Migraine headache in perimenopausal and menopausal women," Current Pain and Headache Reports, vol. 13, no. 5, pp. 399-403, 2009.

[4] J. G. Greene, "A factor analytic study of climacteric symptoms," Journal of Psychosomatic Research, vol. 20, no. 5, pp. 425-430, 1976.

[5] M. Hunter, R. Battersby, and M. Whitehead, "Relationships between psychological symptoms, somatic complaints and menopausal status," Maturitas, vol. 8, no. 3, pp. 217-228, 1986.

[6] J. Zwart, G. Dyb, K. Hagen et al., "Depression and anxiety disorders associated with headache frequency. The Nord-Trøndelag Health Study," European Journal of Neurology, vol. 10, no. 2, pp. 147-152, 2003.

[7] J. P. Brown, L. Gallicchio, J. A. Flaws, and J. K. Tracy, "Relations among menopausal symptoms, sleep disturbance and depressive symptoms in midlife," Maturitas, vol. 62, no. 2, pp. 184-189, 2009.

[8] M. Terauchi, S. Hiramitsu, M. Akiyoshi et al., "Associations among depression, anxiety, and somatic symptoms in peri- and postmenopausal women," Journal of Obstetrics and Gynaecology Research, vol. 39, no. 5, pp. 1007-1013, 2013.

[9] F. Yu, T. Takahashi, J. Moriya et al., "Traditional Chinese medicine and kampo: a review from the distant past for the future," Journal of International Medical Research, vol. 34, no. 3, pp. 231-239, 2006.

[10] V. Scheid, T. Ward, W. Cha, K. Watanabe, and X. Liao, “The treatment of menopausal symptoms by traditional East Asian medicines: review and perspectives," Maturitas, vol. 66, no. 2, pp. 111-130, 2010.

[11] M. Terauchi, S. Obayashi, M. Akiyoshi, K. Kato, E. Matsushima, and T. Kubota, "Insomnia in Japanese peri-and postmenopausal women," Climacteric, vol. 13, no. 5, pp. 479-486, 2010.

[12] M. Terauchi, S. Obayashi, M. Akiyoshi, K. Kato, E. Matsushima, and T. Kubota, "Effects of oral estrogen and hypnotics on Japanese periand postmenopausal women with sleep disturbance," Journal of Obstetrics and Gynaecology Research, vol. 37, no. 7, pp. 741-749, 2011.

[13] M. Terauchi, S. Hiramitsu, M. Akiyoshi et al., "Effects of three Kampo formulae: Tokishakuyakusan (TJ-23), Kamishoyosan (TJ-24), and Keishibukuryogan (TJ-25) on Japanese peri- and postmenopausal women with sleep disturbances," Archives of Gynecology and Obstetrics, vol. 284, no. 4, pp. 913-921, 2011.

[14] M. Terauchi, M. Akiyoshi, Y. Owa, K. Kato, S. Obayashi, and T. Kubota, "Effects of the Kampo medication keishibukuryogan on blood pressure in perimenopausal and postmenopausal women," International Journal of Gynecology and Obstetrics, vol. 114, no. 2, pp. 149-152, 2011.

[15] M. Terauchi, S. Hiramitsu, M. Akiyoshi et al., "Effects of three Kampo formulae: tokishakuyakusan (TJ-23), Kamishoyosan (TJ-24), and Keishibukuryogan (TJ-25) on Japanese peri- and postmenopausal women with sleep disturbances," Archives of Gynecology and Obstetrics, vol. 284, no. 4, pp. 913-921, 2011. 
[16] M. Terauchi, S. Hiramitsu, M. Akiyoshi et al., "Associations between anxiety, depression and insomnia in peri- and postmenopausal women," Maturitas, vol. 72, no. 1, pp. 61-65, 2012.

[17] H. M. Kravitz, P. A. Ganz, J. Bromberger, L. H. Powell, K. SuttonTyrrell, and P. M. Meyer, "Sleep difficulty in women at midlife: a community survey of sleep and the menopausal transition," Menopause, vol. 10, no. 1, pp. 19-28, 2003.

[18] M. Hunter, "The South-East England longitudinal study of the climacteric and postmenopause," Maturitas, vol. 14, no. 2, pp. 117-126, 1992.

[19] T. Yambe, M. Yoshizawa, Y. Saijo et al., "Brachio-ankle pulse wave velocity and cardio-ankle vascular index (CAVI)," Biomedicine and Pharmacotherapy, vol. 58, supplement 1, pp. S95-S98, 2004.

[20] B. L. Neugarten and R. J. Kraines, “'Menopausal Symptoms', in women of various ages," Psychosomatic Medicine, vol. 27, no. 3, pp. 266-273, 1965.

[21] J. Berecki-Gisolf, N. Begum, and A. J. Dobson, "Symptoms reported by women in midlife: menopausal transition or aging?" Menopause, vol. 16, no. 5, pp. 1021-1029, 2009.

[22] K. Kroenke, R. L. Spitzer, and J. B. W. Williams, "The PHQ-15: validity of a new measure for evaluating the severity of somatic symptoms," Psychosomatic Medicine, vol. 64, no. 2, pp. 258-266, 2002.

[23] I. Neri, F. Granella, R. Nappi, G. C. Manzoni, F. Facchinetti, and A. R. Genazzani, "Characteristics of headache at menopause: a clinico-epidemiologic study," Maturitas, vol. 17, no. 1, pp. 31-37, 1993.

[24] E. W. Freeman, M. D. Sammel, H. Lin, C. R. Gracia, and S. Kapoor, "Symptoms in the menopausal transition: hormone and behavioral correlates," Obstetrics and Gynecology, vol. 111, no. 1, pp. 127-136, 2008.

[25] K. L. Aegidius, J.-A. Zwart, K. Hagen, B. Schei, and L. J. Stovner, "Hormone replacement therapy and headache prevalence in postmenopausal women. The Head-HUNT study," European Journal of Neurology, vol. 14, no. 1, pp. 73-78, 2007.

[26] L. Mueller, "Predictability of exogenous hormone effect on subgroups of migraineurs," Headache, vol. 40, no. 3, pp. 189-193, 2000.

[27] K. M. Brett and N. L. Keenan, "Complementary and alternative medicine use among midlife women for reasons including menopause in the United States: 2002," Menopause, vol. 14, no. 2, pp. 300-307, 2007.

[28] C. P. van der Sluijs, A. Bensoussan, L. Liyanage, and S. Shah, "Women's health during mid-life survey: the use of complementary and alternative medicine by symptomatic women transitioning through menopause in Sydney," Menopause, vol. 14, no. 3, pp. 397-403, 2007.

[29] B. Ettinger, S. M. Wang, R. S. Leslie et al., "Evolution of postmenopausal hormone therapy between 2002 and 2009," Menopause, vol. 19, no. 6, pp. 610-615, 2012.

[30] B. L. Sprague, A. Trentham-Dietz, and K. A. Cronin, "A sustained decline in postmenopausal hormone use: results from the National Health and Nutrition Examination Survey, 19992010," Obstetrics \& Gynecology, vol. 120, no. 3, pp. 595-603, 2012.

[31] J. Adams, G. Barbery, and C. W. Lui, "Complementary and alternative medicine use for headache and migraine: a critical review of the literature," Headache, vol. 53, no. 3, pp. 459-473, 2012.

[32] C. Peng, X. Xie, L. Wang, L. Guo, and T. Hu, "Pharmacodynamic action and mechanism of volatile oil from Rhizoma Ligustici
Chuanxiong Hort. on treating headache," Phytomedicine, vol. 16, no. 1, pp. 25-34, 2009.

[33] Y. Wang, S. Liang, D. Xu et al., "Effect and mechanism of senkyunolide $\mathrm{i}$ as an anti-migraine compound from Ligusticum chuanxiong," Journal of Pharmacy and Pharmacology, vol. 63, no. 2, pp. 261-266, 2011.

[34] F. Xu, D. Peng, C. Tao et al., "Anti-depression effects of Danggui-Shaoyao-San, a fixed combination of Traditional Chinese Medicine, on depression model in mice and rats," Phytomedicine, vol. 18, no. 13, pp. 1130-1136, 2011.

[35] N. O’Flynn and L. Ridsdale, "Headache in primary care: how important is diagnosis to management?" British Journal of General Practice, vol. 52, no. 480, pp. 569-573, 2002. 


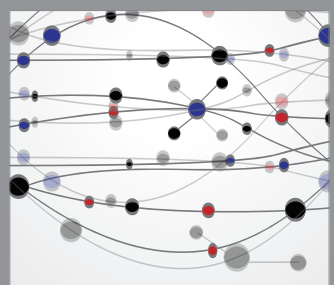

The Scientific World Journal
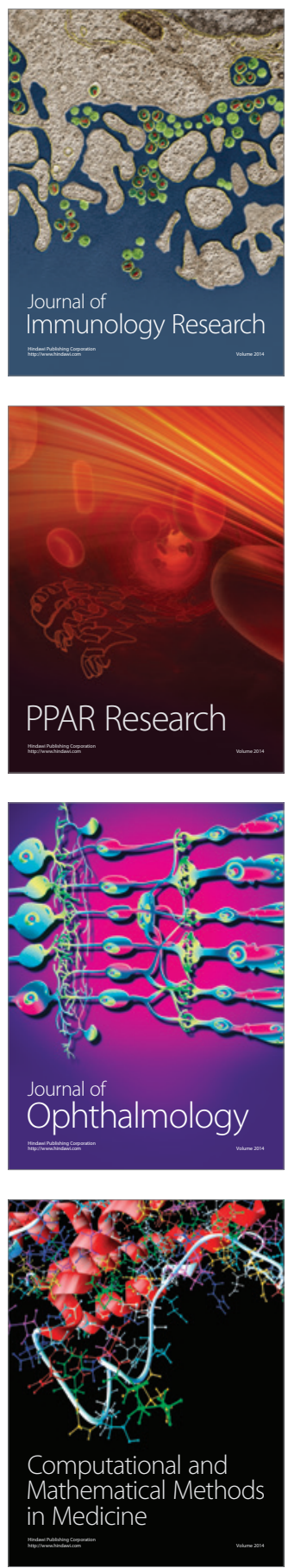

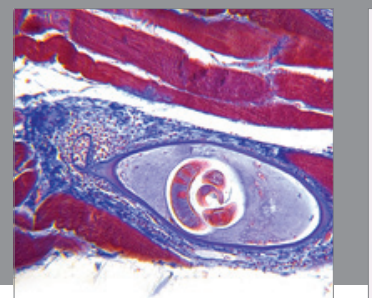

Gastroenterology

Research and Practice
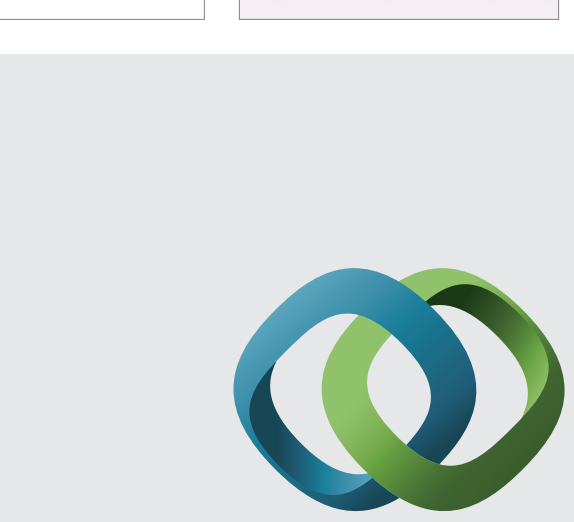

\section{Hindawi}

Submit your manuscripts at

http://www.hindawi.com
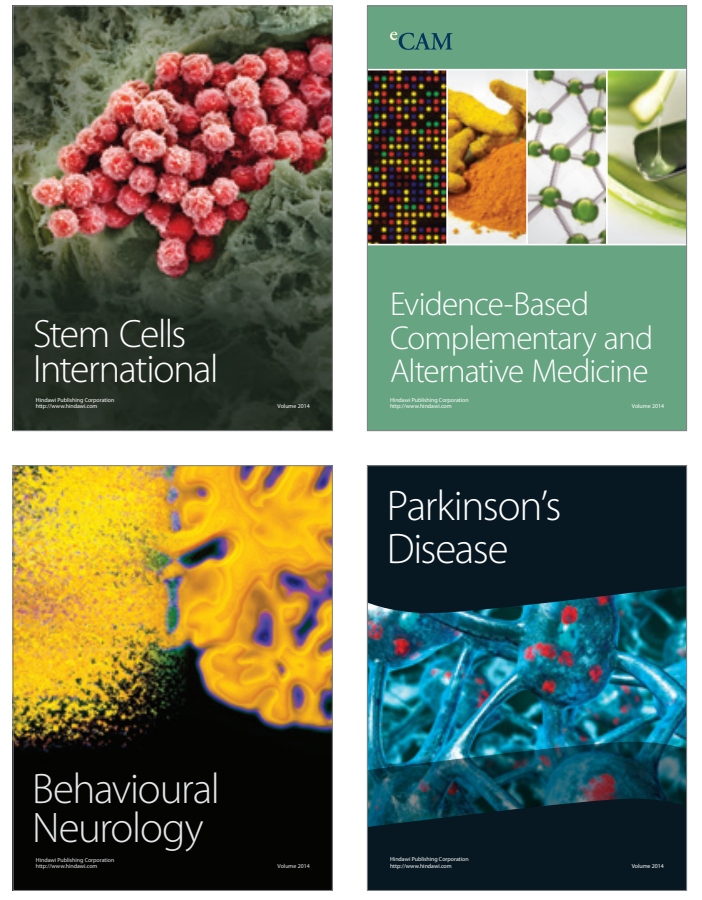
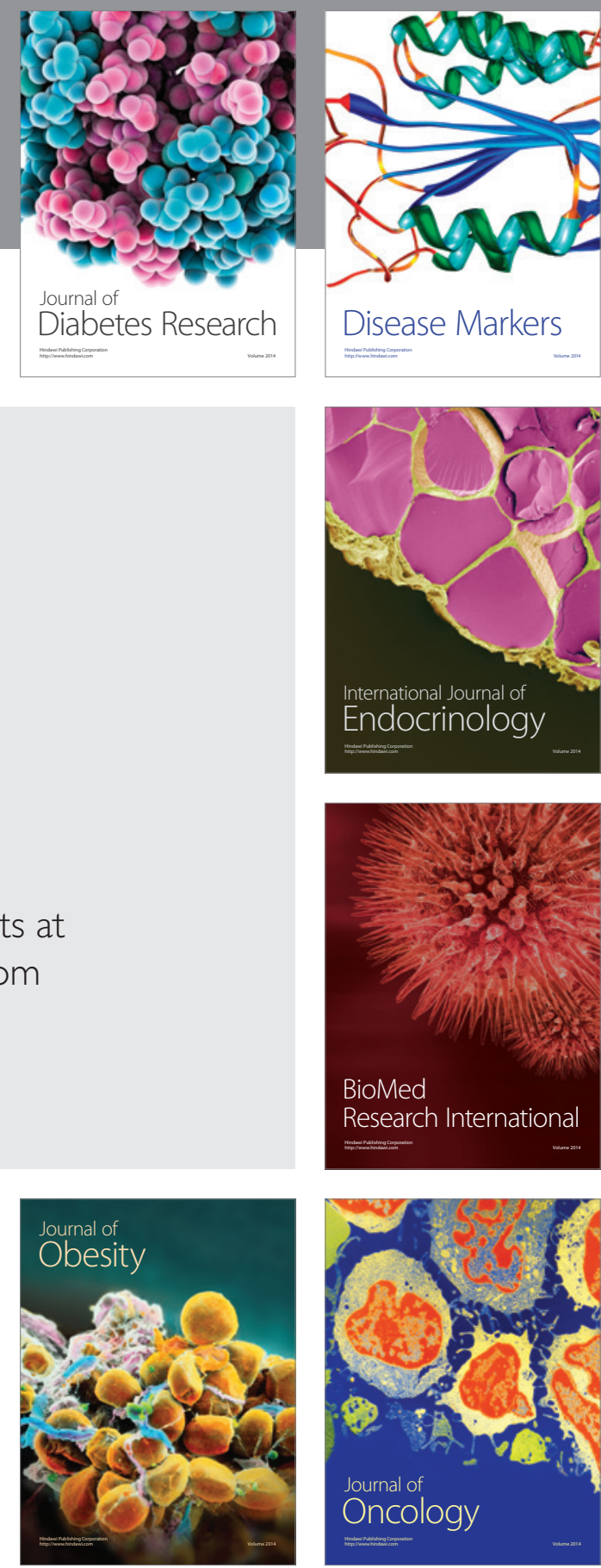

Disease Markers
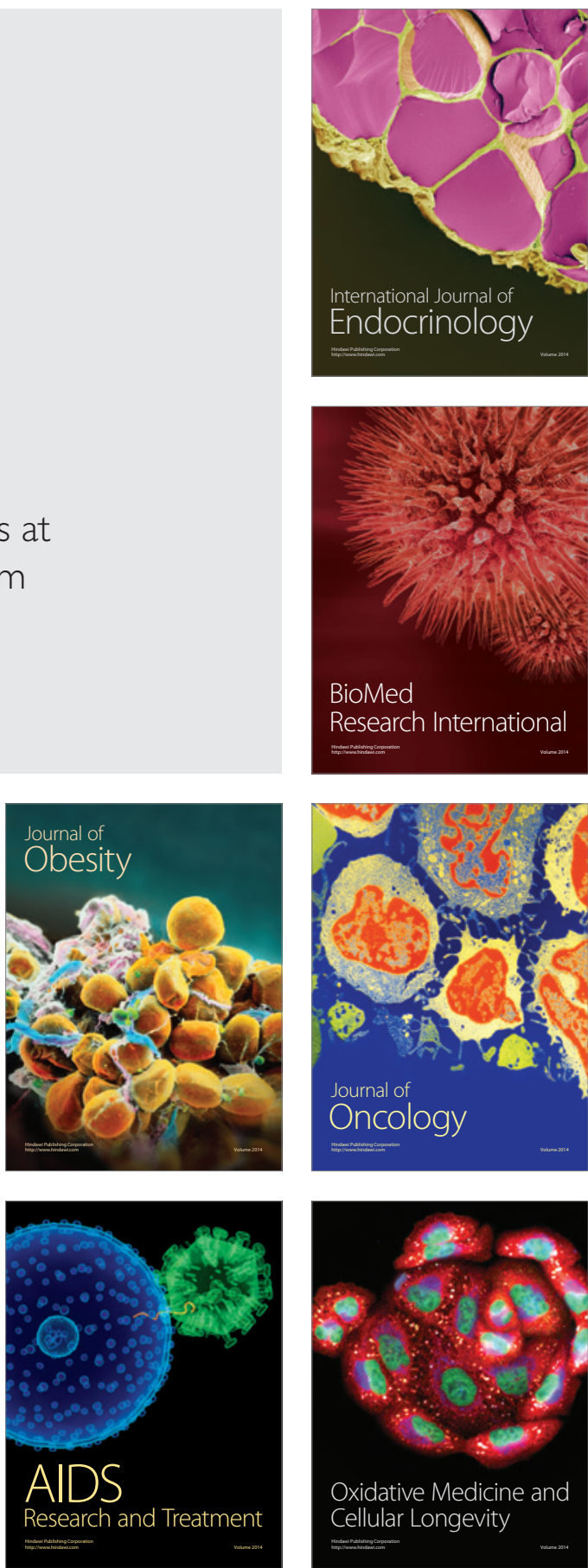